\title{
25 Research Soure \\ Prevalence Of Malaria; A Retrospective Study On The Trend Of Malaria From 2015 To 2021 In A Tertiary Care Centre, Puducherry
}

\section{Rachana Kannambath}

JIPMER: Jawaharlal Institute of Postgraduate Medical Education and Research

Nonika Rajkumari ( $\nabla$ nonika.raj@gmail.com )

Jawaharlal Institute of Postgraduate Medical Education and Research https://orcid.org/0000-0002-4326-3877

Monika Sivaradjy

JIPMER: Jawaharlal Institute of Postgraduate Medical Education and Research

\section{Research Article}

Keywords: Malaria, Prevalence, Plasmodium, India

Posted Date: February 23rd, 2022

DOI: https://doi.org/10.21203/rs.3.rs-1361629/v1

License: (c) (i) This work is licensed under a Creative Commons Attribution 4.0 International License. Read Full License 


\section{Abstract}

Battle against malaria has been going on since time immemorial. Understanding the true burden of disease and the determinants of its transmission are important for implementing adequate control measures. This study intends to explore the local epidemiology and burden of malaria in Puducherry, a coastal Union territory located in Southern part of India over a period of 7 years.A retrospective record-based study was conducted from 2015 to 2021, where details from all samples tested positive for malaria by peripheral blood examination or rapid card test, from suspected cases were collected and analyzed. The overall prevalence of malaria over the 7 years was $1.7 \%(257 / 14888)$. Majority of the patients were males (75.88\%) and the major age group affected was from 21 to 40 years (56.03\%). Disease was maximum seen during the monsoon season followed by the post monsoon season. Vivax malaria predominated irrespective of the gender, seasonal change and different age groups except in children < 10 years were both falciparum and vivax malaria were seen in equivalence. Major species to cause infection among infants were Plasmodium falciparum (3/4). This study shows a declining trend of malaria transmission over the years. There is no change in the predominant species affected or seasonal trends over the years. The possibility of underestimation of cases due to various factors cannot be ignored.

\section{Introduction}

Malaria is a major public health problem all over the world. It is caused by a protozoan parasite Plasmodium species of which 5 major species are known to cause human infection such as, Plasmodium falciparum (P. falciparum), Plasmodium vivax (P. vivax), Plasmodium malariae ( $P$. malariae), Plasmodium ovale ( $P$. ovale) and Plasmodium knowlesi (P. knowlesi) (Talapko et al.2019). Malaria is known to be a human disease since time immemorial. The first description of the disease was found in the Chinese medical records of $2700 \mathrm{BC}$. In the ancient times, the illness was attributed to supernatural forces and angry deities, followed by the belief that it is caused by inhalation of vapors from the swamp (Talapko et al.2019). It was a French army doctor Charles Louis Alphonse Laveran, who discovered malaria parasites in the blood of infected patients(1880), for which he was awarded Nobel prize. Later in 1897, Surgeon-Major Ronald Ross of British Indian Medical service discovered the life cycle of malarial parasites inside the mosquito (Arrow et al.2004). Since then the fight against malaria has started, which led to various practical proposals for the interruption of transmission, appropriate diagnosis and treatment of malaria, which witnessed several ups and downs. At present, according to the latest malaria report of 2021, there are 85 malaria endemic countries, with a 241 million cases in the year of 2021 globally. In this 95\% of the burden is accounted from the WHO African region. WHO South East Asian countries accounted for $2 \%$ of the global burden, among which $83 \%$ cases are contributed from India. (WHO World malaria report 2021) The trend of malaria in India has shown a lot of variations. India witnessed peak of malaria in 1950's with a burden of 75 million cases and 0.8 million per year mortality. The National Malaria Control Program came in to force in 1953 , following which there was a massive reduction of cases to less than 50,000 with no mortality (1961). Malaria was thought to be at the verge of eradication, but it made a comeback with 6.45 million cases in 1976(Anvikar et al.2016). Various factors are thought to have caused this resurgence such as, urbanization and subsequent favorable environment for the breeding of the vector, disruption of the control program due to war or conflicts in the country, emergence of resistance to insecticides among the vectors, drug resistant parasites etc. (Nasir et al.2020). Modified plan of action was introduced in 1977 which was not of much benefit, reintroduced in mid-1980's followed by in 1995. Since then the trend of malaria has shown a gradual decrease in India. Currently the National Framework for Malaria Elimination introduced in 2016, intends to interrupt the transmission of indigenous cases and achieve malaria free certificate by 2030 (Nasir et al.2020). The two major species causing malaria in India are $P$. falciparum and $P$. vivax and their distribution varies from place to place (Das et al.2012). There are no many studies exploring the local epidemiology and burden of malaria in South India, especially in Puducherry. This study intends to look at the trend of malaria in Puducherry, a coastal Union territory in the Southern part of India, over a period of 7

Page $2 / 10$ 
years, the distribution of Plasmodium species over the years and the influence of demographic characters and climate to the disease.

\section{Methodology}

A retrospective record based observational study was conducted in the department of Microbiology, of a tertiary care health centre, Puducherry. All the blood samples which were positive for malaria by any of the diagnostic method, from 2015 to 2021 were included in the study. Testing for malaria was done on the request of the clinicians, from suspected cases based on history and clinical examination. All the received samples were subjected to immunochromatographic test detecting the Plasmodium spp. antigen (lactate dehydrogenase/aldolase) and Plasmodium falciparum specific antigen (Histidine rich protein 2) and peripheral blood examination by thin smear, thick smear, quantitative buffy coat examination. All these were screened by two independent persons before confirmation. In the last three years of the study period, an automated instrument Parasight ${ }^{\mathrm{TM}}$ platform was used in addition to the above-mentioned methods.

\section{Results}

A total of 14,888 samples were received over the 7year study period from malaria suspected cases, of which 257 samples (1.73\%) were tested positive for malaria by either/both the laboratory diagnostic method used. Among this majority were $P$. vivax (161/257,62.65\%), followed by $P$. falciparum (80/257,31.13\%). Species identification could not be done in 16 samples (6.23\%). Out of the 257 malaria positive samples, 215 were positive by both rapid card test as well as peripheral blood examination by conventional microscopy or automated Parasight ${ }^{\text {TM }}$ platform, 38 samples were positive only by rapid card test, out of which 22 were positive for the HRP 2 antigen, thereby reported as $P$. falciparum. Four samples were negative by rapid card test, but positive for $P$. falciparum by peripheral blood examination. The year wise distribution of the total number of samples tested, total number of positives and the Plasmodium species distribution of each year are depicted in Table 1.Males(75.88\%) were predominantly affected, with a male to female ratio of 3.15.The age of the patients ranged from 3 months to 75 years, with the major age group affected being 21 to 40 years(144/257,56.03\%). Extremes of ages were comparatively less affected,14/257(5.45\%) were children $\leq 10$ years, among which 10 children were less than 5 years old. Four among them were infants. Elderly population ( $>60$ years) affected were also meagre,11/257(4\%). The trend of occurrence of malaria cases based on the age and gender over the years are depicted in Table 2 and Fig. 1 respectively. Distribution of different Plasmodium species based on various age groups and gender are depicted in Table 3. Though $P$. vivax was the predominant species affecting all the age groups, in consistence with the general trend but, equivalent number of $P$, falciparum was observed among the children ( $\leq 10$ years). Among the 4 malaria positive infants, 3 were caused by $P$. falciparum and one by unidentified non falciparum species. 
Table 1

The year wise distribution of the total number of samples tested, total number of malaria positive samples and the Plasmodium species distribution

\begin{tabular}{|llllll|}
\hline Year & $\begin{array}{l}\text { Total no of samples } \\
\text { received }\end{array}$ & $\begin{array}{l}\text { Total } \\
\text { positives }\end{array}$ & $\begin{array}{l}\text { P } \\
\text { falciparum }\end{array}$ & P. vivax & $\begin{array}{l}\text { Unidentified Plasmodium } \\
\text { species }\end{array}$ \\
\hline 2015 & 1483 & $22(1.48 \%)$ & $8(36.36 \%)$ & $13(59.09 \%)$ & $1(4.54 \%)$ \\
\hline 2016 & 1675 & $41(2.45 \%)$ & $15(36.58 \%)$ & $23(56.1 \%)$ & $3(7.32 \%)$ \\
\hline 2017 & 4096 & $89(2.17 \%)$ & $29(32.58 \%)$ & $55(61.8 \%)$ & $5(5.62 \%)$ \\
\hline 2018 & 2074 & $62(2.99 \%)$ & $14(22.58 \%)$ & $43(69.35 \%)$ & $5(8.06 \%)$ \\
\hline 2019 & 2831 & $30(1.06 \%)$ & $10(33.33 \%)$ & $18(60 \%)$ & $2(6.67 \%)$ \\
\hline 2020 & 1024 & $3(0.29 \%)$ & $1(33.33 \%)$ & $2(66.67 \%)$ & 0 \\
\hline 2021 & 1705 & $10(0.59 \%)$ & $3(30 \%)$ & $7(70 \%)$ & 0 \\
\hline Total & 14888 & $257(1.73 \%)$ & $80(31.13 \%)$ & $161(62.64 \%)$ & $16(6.22 \%)$ \\
\hline
\end{tabular}

Table 2

Age group wise distribution of the malaria cases over the years

\begin{tabular}{|llllllllll|}
\hline $\begin{array}{l}\text { Year } \\
\text { Age }\end{array}$ & $\leq 10$ & $\mathbf{1 1 - 2 0}$ & $\mathbf{2 1 - 3 0}$ & $\mathbf{3 1 - 4 0}$ & $\mathbf{4 1 - 5 0}$ & $\mathbf{5 1 - 6 0}$ & $\mathbf{6 1 - 7 0}$ & $\mathbf{2} 71$ & Total \\
\hline 2015 & $2(9.1 \%)$ & $5(22.7 \%)$ & $7(31.8 \%)$ & $4(18.2 \%)$ & $2(9.1 \%)$ & $1(4.5 \%)$ & $1(4.5 \%)$ & 0 & 22 \\
\hline 2016 & $2(4.9 \%)$ & $6(14.6 \%)$ & $13(31.7 \%)$ & $8(19.5 \%)$ & $5(12.2 \%)$ & $4(9.8 \%)$ & $2(4.9 \%)$ & $1(2.4 \%)$ & 41 \\
\hline 2017 & $3(3.4 \%)$ & $8(9 \%)$ & $40(44.9 \%)$ & $17(19.1 \%)$ & $11(12.4 \%)$ & $8(9 \%)$ & $2(2.2 \%)$ & 0 & 89 \\
\hline 2018 & $4(6.4 \%)$ & $7(11.3 \%)$ & $22(35.4 \%)$ & $17(27.4 \%)$ & $8(12.9 \%)$ & $1(1.6 \%)$ & $2(3.2 \%)$ & $1(1.6 \mid \%)$ & 62 \\
\hline 2019 & $2(6.7 \%)$ & $5(16.7 \%)$ & $7(23.3 \%)$ & $5(16.7 \%)$ & $4(13.3 \%)$ & $5(16.7 \%)$ & $2(6.7 \%)$ & 0 & 30 \\
\hline 2020 & $1(3.3 \%)$ & 0 & $1(33.3 \%)$ & $1(33.3 \%)$ & 0 & 0 & 0 & 0 & 3 \\
\hline 2021 & 0 & $2(20 \%)$ & $4(40 \%)$ & $2(20 \%)$ & 0 & $2(20 \%)$ & 0 & 0 & 10 \\
\hline Total & $14(5.4 \%)$ & $33(12.8 \%)$ & $94(36.6 \%)$ & $54(21 \%)$ & $30(11.7 \%)$ & $21(8.2 \%)$ & $9(3.5 \%)$ & $2(0.8 \%)$ & 257 \\
\hline
\end{tabular}


Table 3

Distribution of Plasmodium species based on the different age groups and gender

\begin{tabular}{|lllll|}
\hline \multicolumn{4}{|l}{ Age group wise species distribution } \\
\hline Age group/Plasmodium spp. & P. falciparum & P. vivax & Plasmodium species & Total \\
\hline$\leq 10$ & $6(42.86 \%)$ & $6(42.86 \%)$ & $2(14.29 \%)$ & $14(100 \%)$ \\
\hline $11-20$ & $11(33.33 \%)$ & $19(57.58 \%)$ & $3(9.09 \%)$ & $33(100 \%)$ \\
\hline $21-30$ & $25(26.6 \%)$ & $64(68.09 \%)$ & $5(5.32 \%)$ & $94(100 \%)$ \\
\hline $31-40$ & $15(27.78 \%)$ & $36(66.67 \%)$ & $3(5.56 \%)$ & $54(100 \%)$ \\
\hline $41-50$ & $12(40 \%)$ & $17(56.67 \%)$ & $1(3.33 \%)$ & $30(100 \%)$ \\
\hline $51-60$ & $10(47.62 \%)$ & $11(52.38 \%)$ & 0 & $21(100 \%)$ \\
\hline $61-70$ & $1(11.11 \%)$ & $6(66.67 \%)$ & $2(22.22 \%)$ & $9(100 \%)$ \\
\hline$\geq 71$ & 0 & $2(100 \%)$ & 0 & $2(100 \%)$ \\
\hline Total & $80(31.13 \%)$ & $161(62.65 \%)$ & $16(6.22 \%)$ & $257(100 \%)$ \\
\hline Gender wise species distribution $)$ & & & Total \\
\hline Gender / Plasmodium spp. & P. falciparum & P. vivax & Plasmodium species & $195(100 \%)$ \\
\hline Male & $65(33.33 \%)$ & $120(61.54 \%)$ & $10(5.13 \%)$ & $62(100 \%)$ \\
\hline Female & $15(24.19 \%)$ & $41(66.13 \%)$ & $6(9.68 \%)$ & $257(100 \%)$ \\
\hline Total & $80(31.13 \%)$ & $161(62.65 \%)$ & $16(6.23 \%)$ & \\
\hline PF-P. falciparum, PV-P. vivax, PS- Plasmodium species & & \\
\hline
\end{tabular}

The prevalence of occurrence of malaria cases over different seasons of a year were analysed. Maximum number of cases were observed in the monsoon season (June to September) followed by the post monsoon season (OctoberNovember). The seasonal trend of occurrence of malaria cases each year has been depicted in Fig. 2. The distribution of the various Plasmodium species over the different seasons were analysed and depicted in the Fig. 3.

\section{Discussion}

Malaria is a major vector borne disease in India for the past several years. India has witnessed a tremendous decline in the number of malaria cases over the past two decades. There was a decline of $71.8 \%$ of cases and $73.9 \%$ drop in mortality over the years 2000 to 2019 (Mohan et al.2021). In spite, India remains the greatest contributor of cases in the WHO South East Asian region (WHO World malaria report 2021). The diverse geography, human genetic diversity and climatic conditions in India has been advantageous for the survival of parasites and its vectors(Singh et al.2009). India possesses a diverse malaria vector habitat of approximately 58 species of anopheline mosquitoes. (Das et al.2012). The trend of malaria prevalence has shown a fluctuating course over the years in our study. There was an increase in burden from 2015 to 2018 followed by a decline in 2019.A tremendous decline is observed in the year of 2020, but this could be attributed to the effect of COVID 19 pandemic and the subsequent lockdown in the country. As per the latest malaria report of 2021 by WHO, the incidence of malaria has increased globally from 56 in 2019 to 59 in 2020(World malaria report 2021, WHO), contrary to the results of this study. This increase in incidence could be attributed to the interruption of health care services which were skewed towards the diagnosis, treatment and control of the COVID 19 pandemic. But the Indian statistics shows that, there is a decline of $45.08 \%$ of cases in 2020 compared to 2019 , 
contrary to the global scenario. Overlapping symptoms of Covid 19 infection and malaria could have led to underreporting of cases which resulted in this low number of cases (Mohan et al.2021). In a systematic review and meta-analysis done to estimate the prevalence of malaria in Covid 19 infected patients, has shown a pooled prevalence of $11 \%$ co-infection between Covid 19 and Plasmodium spp. Majority of the cases were reported from Nigeria followed by India (Wilairatana et al.2021). Moreover, malaria and Covid 19 co-infections are found to be associated with severe degree of coagulopathy and severe disease, given the procoagulant state contributed by both the infections (Hussein et al.2020). Another interesting feature observed is low prevalence of Covid 19 in malaria high endemic areas such as African countries, where Covid transmission is considered to be relatively less. Variable factors like, polymorphisms associated with angiotensin converting enzyme 1 and 2 in malaria patients, which also act as a receptor for Covid 19 virus, anti GPI antibodies produced in malaria patients conferring protection against Covid 19 virus, prophylactic and therapeutic effect of hydroxychloroquine and chloroquine on Covid 19 infections are proposed to be the reason (Hussein et al.2020). In our study, there was no co-infection with Covid 19 infection detected in any of the malaria positive patients.

Plasmodium species distribution varies greatly from place to place in India. $P$. falciparum and $P$. vivax are the two most common species known to cause malaria in India, followed by $P$. malariae reported mainly from Eastern part of India. Reports of $P$. ovale are rare and $P$. knowlesi even rarer (Das et al.2012). $P$. vivax was the predominant species historically, but the incidence of vivax malaria had shown a decreasing trend in India. ${ }^{3}$ In 1985 the ratio of P.falciparum to P.vivax was 0.41 which increased to 1.01 in 2010 . Still, $47 \%$ of vivax malaria cases reported globally are from India (Nasir et al.2020). In our study the overall ratio of $P$. falciparum to $P$. vivax is 0.49 (Das et al.2012). Identification of Plasmodium species is important from the treatment point of view as well as the implementation of control measures. In addition, $P$. vivax which was earlier thought to cause benign malaria are now known to cause serious manifestations as well (Kojom et al.2021). The frequent relapses encountered due to the persistence of hypnozoites also poses a challenge in therapy as well as control programmes (Ghosh et al.2019). Other than $P$. falciparum and $P$. vivax, no other species or mixed infections were detected in our study. The use of only conventional methods for the diagnosis of malaria during most of the study period could be a reason for this. In a study conducted in a high malaria burden state in India has shown that the sensitivity of detection of $P$. falciparum is $>80 \%$ using peripheral blood smear examination and rapid diagnostic test compared to molecular method, but for the identification of vivax malaria as well as mixed infections, the sensitivity was very poor using non molecular methods-57\% and 18\% respectively (Ahmad et al.2021).

On analysing the age and gender distribution of malaria, majority of the population affected were young adult males overall as well as in each year. This is in concordance with other reports (Dayanand et al.2017, Pradhan et al.2022, Gupta et al.2009). The male preponderance of infection could be due to various factors like, difference in vector exposure risk, different travel habits, distinction in mosquito attraction, hormonal or host genetic factors etc (Van Ejik et al.2019). Children are considered to be the most vulnerable population owing to their immature immune system and are prone to develop severe malaria and subsequent developmental and cognitive impairment (Abossie et al.2020). Young infants less than 6 months of age are at lesser risk, due to the protective maternal antibodies passed on. Six months to 5 -year children are considered to be at maximum risk of developing severe malaria as well as its complications (Philips et al.2017). But in our study, only 3.5\% of the affected population consisted of children of age 6 months to 5 years. On analyzing the species distribution gender wise, there was no variation from the general trend. But the distribution of $P$. vivax and and $P$. falciparum was found to be equal among children less than 10 years. The causative agents in majority of the infants were $P$. falciparum.

Vector borne diseases are dependent on climate variations. Therefore, weather factors such as optimum rainfall, temperature, humidity etc are important determinants of malaria transmission (Kumar et al.2022). The minimum temperature beyond which $P$. vivax fails to develop inside the anopheline mosquito is $14.5-16.5$ degree Celsius, 
whereas for $P$. falciparum it is 16.5 to 19 degrees Celsius. But as the temperature rises to 20 to 30 degrees Celsius, the duration of lifecycle of the Plasmodium spp. inside the mosquito decreases. Similarly at this temperature, rate of blood digestion as well as reproductive development of the vector also occurs in a faster pace resulting in frequent blood meals and wide transmission of infection. But if the temperature is increased beyond 32 to 39 degrees Celsius, it is detrimental to the survival of mosquito (Kumar et al.2022). Likewise, moderate amount of rainfall is beneficial to the breeding of mosquitoes where as heavy rainfall/flooding can lead to washing away of the mosquito larva (Chaturvedi et al.2021). These factors vary from place to place. Therefore, it is essential to understand the malaria transmission dynamics according to seasons or climatic conditions of a particular area, which will help in anticipating the burden of cases and to adopt appropriate control measures. In our study peak of malaria cases were noted in the monsoon season in all the years except in 2020, where the data may be a misrepresentation of the actual burden due to the Covid pandemic. This was in concordance with many other studies from India (Mopuri et al.2021, Kessler et al.2018). In a study conducted in New Delhi, it was noted that $P$. falciparum malaria peaked during the post monsoon season, whereas $P$. vivax peaked during monsoon season (Gupta et al.2009). However, in our study, there was no such distinction observed with respect to the difference in species.

Knowledge on the precise burden of the disease in each region, as well as the understanding of the determinants of disease risk is important to reach the goal of malaria elimination. Even though, India has attained progress in reducing the case burden and mortality in the past two decades, unfortunately huge gap exists between the true case scenario and the one which has been represented. For example, a surveillance-based estimation of malaria burden by a nodal research body in 2015/2016 India, has revealed that the estimated malaria burden in India is four times higher than that reported by the national programme (Rahi et al.2020). Overall, the trend of malaria in our area shows a declining pattern, but the possibility of underreporting of cases cannot be ruled out.

\section{Declarations}

Conflict of interest-All authors declare that they have no conflict of interest

Ethics statement- The study had been approved by the institute ethics committee

\section{Author contribution statement}

RK was responsible for data collection, analysis, literature search, preparation and submission of the manuscript.NR was responsible for conceptualisation, critical analysis of the data, review and editing of the manuscript. MS was involved in data collection and analysis.

\section{References}

1. Abossie A, Yohanes T, Nedu A, Tafesse W, Damitie M (2020) Prevalence of Malaria and Associated Risk Factors Among Febrile Children Under Five Years: A Cross-Sectional Study in Arba Minch Zuria District, South Ethiopia. Infect Drug Resist 13:363-372

2. Ahmad A, Soni P, Kumar L, Singh MP, Verma AK, Sharma A et al (2021) Comparison of polymerase chain reaction, microscopy, and rapid diagnostic test in malaria detection in a high burden state (Odisha) of India. Pathog Glob Health 115:267-272

3. Anvikar AR, Shah N, Dhariwal AC, Sonal GS, Pradhan MM, Ghosh SK et al (2016) Epidemiology of Plasmodium vivax Malaria in India. Am J Trop Med Hyg 95:108-120

4. Arrow KJ, Panosian C, Gelband H (2004) A brief history of malaria. Saving lives, buying time. Economics of malaria drugs in an age of resistance. National Academies Press, Washington, p 125

Page $7 / 10$ 
5. Chaturvedi S, Dwivedi S (2021) Understanding the effect of climate change in the distribution and intensity of malaria transmission over India using a dynamical malaria model. Int J Biometeorol 65:1161-1175

6. Das A, Anvikar AR, Cator LJ, Dhiman RC, Eapen A, Mishra N et al (2012) Malaria in India: the center for the study of complex malaria in India. Acta Trop 121:267-273

7. Dayanand KK, Punnath K, Chandrashekar V, Achur RN, Kakkilaya SB, Ghosh SK et al (2017) Malaria prevalence in Mangaluru city area in the southwestern coastal region of India. Malar J 16:492

8. Ghosh SK, Rahi M (2019) Malaria elimination in India-The way forward. J Vector Borne Dis 56:32-40

9. Gupta S, Gunter JT, Novak RJ, Regens JL (2009) Patterns of Plasmodium vivax and Plasmodium falciparum malaria underscore importance of data collection from private health care facilities in India. Malar J 8:1-8

10. Hussein MIH, Albashir AAD, Elawad OAMA, Homeida A (2020) Malaria and COVID-19: unmasking their ties. Malar J 19:457

11. Kessler A, van Eijk AM, Jamir L, Walton C, Carlton JM, Albert S (2018) Malaria in Meghalaya: a systematic literature review and analysis of data from the National Vector-Borne Disease Control Programme. Malar J 17:411

12. Kojom Foko LP, Arya A, Sharma A, Singh V (2021) Epidemiology and clinical outcomes of severe Plasmodium vivax malaria in India. J Infect 82:231-246

13. Kumar P, Pisudde P, Parth Sarthi P (2022) Meteorological linkage of Malaria cases in the eastern state of India. J Clim Change Health 5:100064

14. Mohan A, Wara UU, Amjad SW, Rackimuthu S, Hunain R, Khan H et al (2021) Malaria amidst COVID-19 in India: Challenges, Efforts, and Recommendations. Clin Epidemiol Glob Health 12:100867

15. Mopuri R, Kakarla SG, Mutheneni SR, Kadiri MR, Kumaraswamy S (2020) Climate based malaria forecasting system for Andhra Pradesh, India. J Parasit Dis 44:497-510

16. Nasir SMI, Amarasekara S, Wickremasinghe R, Fernando D, Udagama P (2020) Prevention of re-establishment of malaria: historical perspective and future prospects. Malar J 19:452

17. Phillips MA, Burrows JN, Manyando C, van Huijsduijnen RH, Van Voorhis WC, Wells TNC (2017) Malaria. Nat Rev Dis Primer 3:17050

18. Pradhan S, Hore S, Maji SK, Manna S, Maity A, Kundu PK, Maity K, Roy S, Mitra S, Dam P, Mondal R (2022) Study of epidemiological behaviour of malaria and its control in the Purulia district of West Bengal, India (2016-2020). Sci Rep 12:1-1

19. Rahi M, Sharma A (2020) For malaria elimination India needs a platform for data integration. BMJ Glob Health 5:e004198

20. Singh V, Mishra N, Awasthi G, Dash AP, Das A (2009) Why is it important to study malaria epidemiology in India? Trends Parasitol 25:452-457

21. Talapko J, Škrlec I, Alebić T, Jukić M, Včev A (2019) Malaria: The Past and the Present. Microorganisms 7:E179

22. Van Eijk AM, Sutton PL, Ramanathapuram L, Sullivan SA, Kanagaraj D, Priya GSL et al (2019) The burden of submicroscopic and asymptomatic malaria in India revealed from epidemiology studies at three varied transmission sites in India. Sci Rep 9:17095

23. World Health Organisation. World Malaria Report

24. Wilairatana P, Masangkay FR, Kotepui KU, Milanez GDJ, Kotepui M (2021) Prevalence and characteristics of malaria among COVID-19 individuals: A systematic review, meta-analysis, and analysis of case reports. PLoS Negl Trop Dis 15:e0009766

\section{Figures}




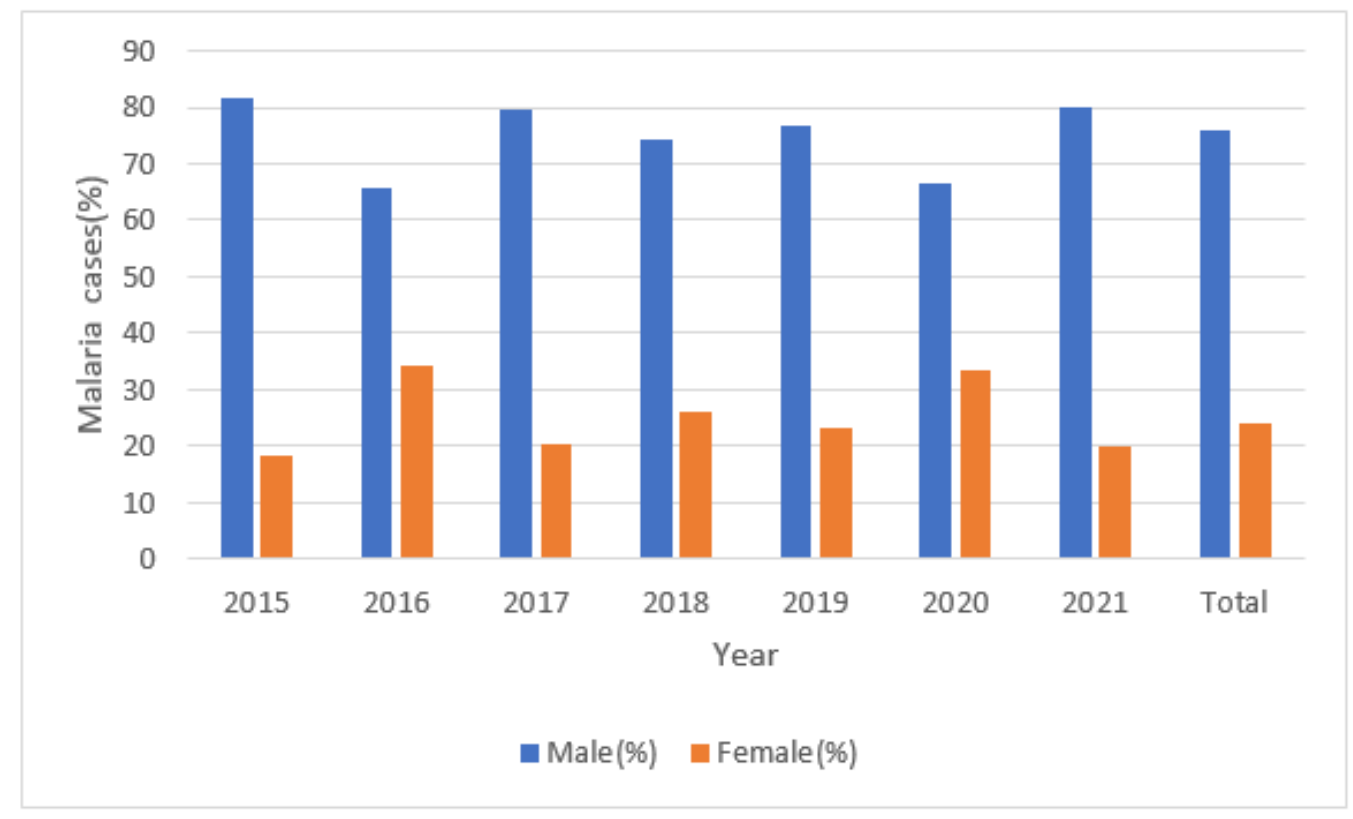

Figure 1

Gender wise distribution of malaria cases over the years

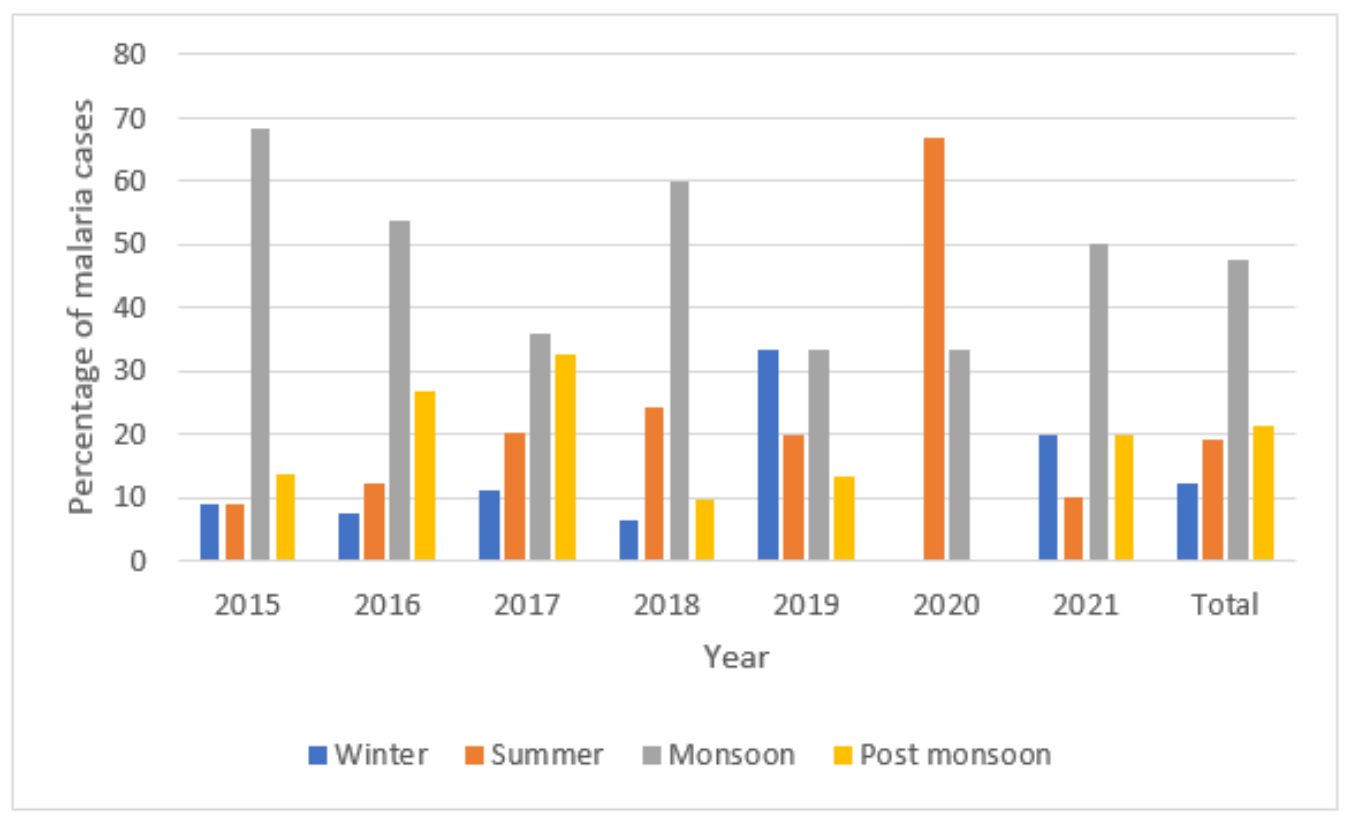

Figure 2

The seasonal trend of malaria over the years 


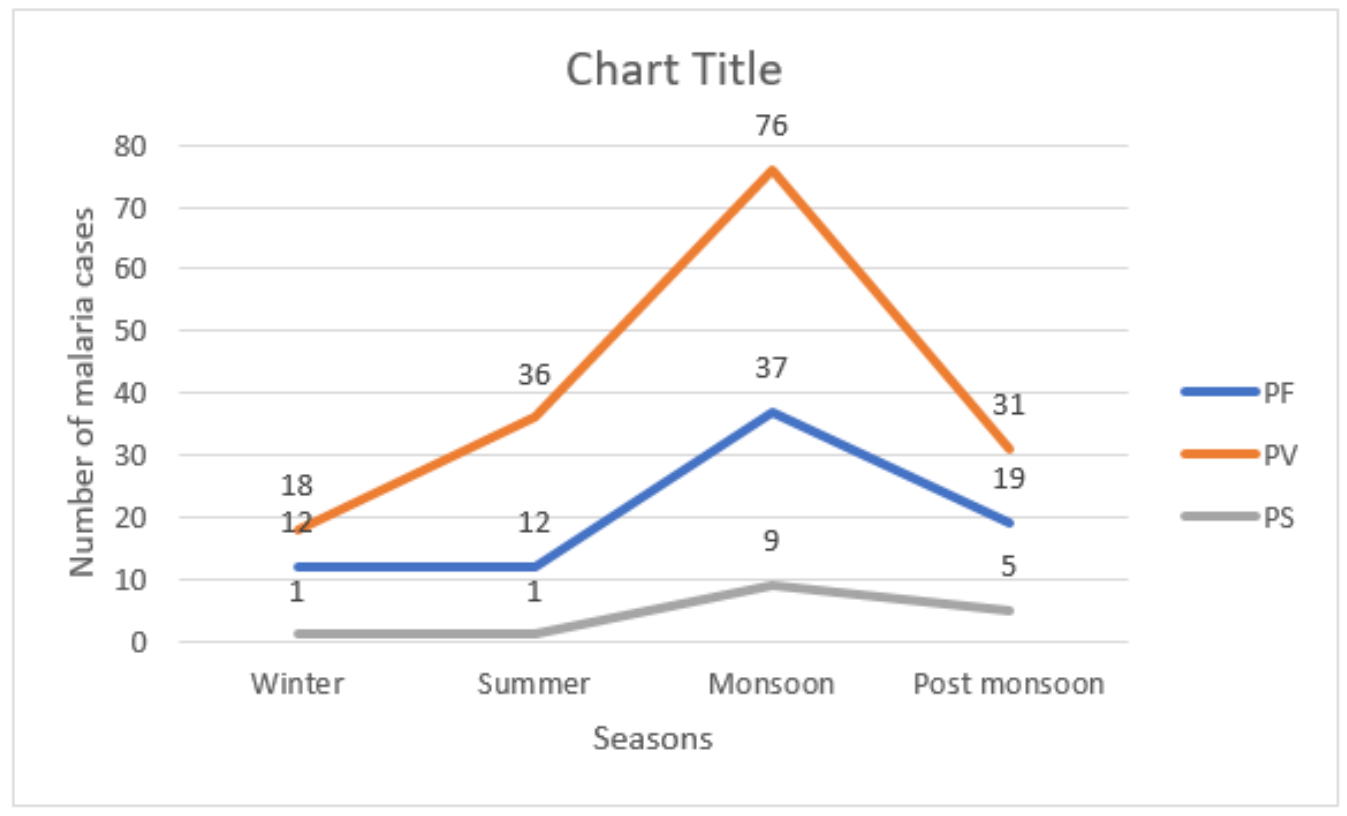

Figure 3

Plasmodium species distribution over the seasons 